'Instituto de Nutrición y Tecnología de los Alimentos (INTA), Universidad de Chile. aMagíster en Planificación del Desarrollo Urbano y Regional. ${ }^{\text {b} A d m i n i s t r a d o r a ~ P u ́ b l i c a . ~}$

Trabajo no recibió financiamiento.

Los autores declaran no tener conflictos de interés.

Recibido el 22 de junio de 2020, aceptado el 10 de mayo de 2021.

Correspondencia a:

Dr. Fernando Vio El Líbano 5524, Santiago de Chile.

fvio@inta.uchile.cl

\section{Evaluación de cursos en línea, masivos y abiertos de educación en salud y nutrición para prevenir la obesidad}

\author{
JUDITH SALINAS ${ }^{1, \mathrm{a},}$, CAROLINA MUÑOZ ${ }^{1, \mathrm{~b}}$, FERNANDO VIO ${ }^{1}$
}

\section{Assessment of massive open online courses for teaching nutrition and preventing obesity}

Background: A massive open online course (MOOC) is an online course aimed at unlimited participation and open access via the Web. Aim: To assess the MOOC experience for teaching nutrition, healthy food consumption, physical activity and health promotion to prevent obesity. Material and Methods: Two MOOC courses, (one with two versions), are described with the registration and evaluation instruments such as surveys carried out at the beginning and at the end of the courses. Effective participants in the three versions were 17.456, 11.121 in MOOC1, 2.351 in MOOC1 second version and 3.984 in MOOC2. Their median age was 31 years, $82 \%$ were women, $60 \%$ were professionals and 12\% were foreigners. Results: In the final evaluation of the three courses, $85 \%$ to $99 \%$ qualified as "very good" or "good" all the surveyed topics. Thirty five percent of participants reported having lack of time, $11 \%$ reported problems with internet connectivity and 3.9\%, personal or work problems. Conclusions: This is the first experience with MOOC in health and nutrition to prevent obesity in Chile. Considering the good results and positive evaluation of these courses, we estimate that they are an important tool to prevent obesity and chronic diseases in Chile, Latin America and other regions of the world.

(Rev Med Chile 2021; 149: 1164-1172)

Key words: Chronic Disease; Education, Distance; Health Education; Obesity.

\footnotetext{
L
}

a obesidad y sobrepeso están aumentando en Chile $^{1}$ y en el mundo ${ }^{2}$. El Estudio NaCional de Educación Física $2015^{3}$ mostró que los estudiantes de octavo año tenían 25\% de sobrepeso y $20 \%$ eran obesos. La Junta Nacional de Auxilio Escolar y Becas (JUNAEB) mostró que la obesidad se triplicó en alumnos de primero año básico entre 1987 y 2019, desde 7,5\% a 24,8\%. En primero medio la obesidad se duplicó entre el 2011 y el 2019 , de $8,2 \%$ a $15,4 \%{ }^{4}$.

Estas cifras confirman el aumento de este problema de salud pública en nuestro país, que afecta mayormente a la población de menores ingresos en un contexto de inequidades ${ }^{5,6}$. La Organización
Mundial de la Salud (OMS) declaró la obesidad como una epidemia global, basado en el aumento del peso corporal en prácticamente todos los países del mundo ${ }^{7}$.

Este aumento es multicausal, pero desde la salud pública existen dos grandes factores que enfrentar: dieta inadecuada y sedentarismo. Ambos componentes han sido objeto de múltiples intervenciones en escuelas desde los años 90, con programas como "Elige Vivir Sano" y leyes como el Etiquetado Nutricional (Ley 20.606), pero que no han tenido impacto en reducir la obesidad ${ }^{8}$. Además, al igual que en todo el mundo, no hay conciencia por parte de la población sobre el pro- 
blema de la obesidad y tampoco de los gobiernos, que no lo consideran un problema prioritario, quedando postergado por temas que aparecen como más urgentes de resolver 9 .

En este contexto y en el marco de los programas de educación continua de promoción de salud del Instituto de Nutrición y Tecnología de los Alimentos (INTA) de la Universidad de Chile desde hace décadas, con metodologías participativas y procesos de evaluación permanente, innovación y mejoramiento de la práctica educativa ${ }^{10-12}$, el año 2017 se incorporan los Cursos Abiertos Masivos en Línea (CAMEL) cuya sigla en inglés es MOOC (Massive Open Online Course).

Estos cursos surgen el 2008 en Canadá y Europa y más recientemente en América Latina con un crecimiento explosivo, fruto del avance tecnológico, la transformación digital de la sociedad y las necesidades de ampliar el acceso a oportunidades de educación a lo largo de la vida ${ }^{13-15}$. Esta popularidad se debe a que prestigiosas universidades se suman a la iniciativa, creando plataformas y modalidades educativas en constante innovación. Los cursos MOOC están basados en una metodología a distancia en entornos abiertos a cualquier persona, sin requisito de escolaridad ni posición social, gratuitos, con énfasis en el proceso de aprendizaje, más que en evaluación y acreditación ${ }^{16,17}$. Entre las tipologías de estos cursos según sus características se encuentran cMOOC, xMOOC, $\mathrm{tMOOC}^{18}$. Los primeros, derivados del enfoque conectivista, están centrados en el significado de la experiencia de aprendizaje, por lo que relevan blogs y herramientas descentralizadas. Los segundos hacen hincapié en el dominio de contenidos y utilizan plataformas como Edx, Coursera o Udciy. Los terceros o tMOOC integran los modelos anteriores acercándose a la perspectiva constructivista, en que el conocimiento se hace a través del refuerzo personal e interacción. Otros autores mencionan transferMOOC para referirse a cursos disponibles que se trasladan a formato MOOC; o bien a cursos MOOC que se insertan en un programa regular o presencial, ampliando el uso de recursos educativos abiertos.

El objetivo de este artículo es evaluar la experiencia de cursos MOOC en el ámbito de la salud y nutrición, en temas de promoción de salud, alimentación saludable y actividad física para prevenir la obesidad, basados en los nuevos modelos de enseñanza aprendizaje a distancia.

\section{Material y Métodos}

\section{Descripción de los Cursos MOOC realizados por el INTA de la Universidad de Chile}

El objetivo general de esta nueva modalidad formativa en el INTA es contribuir a la reflexión sobre los problemas nutricionales y sus causas, como la alimentación, entornos no saludables, la prevención de la obesidad y promoción de estilos de vida saludables, generando diálogo para construir ambientes propicios para la salud y calidad de vida, en especial en escuelas.

Los elementos de esta metodología son:

- Contenidos transmitidos en video-clases cortas (7 a $10 \mathrm{~min}$ ) conducidas por un académico y entrevistas a actores claves, complementados con lecturas y recursos audiovisuales (obligatorios y complementarios).

- Actividades de aprendizaje interactivo, foros, ejercicios y preguntas reflexivas para la acción.

- Proceso evaluativo del aprendizaje y de la satisfacción de los participantes.

- Equipos docentes constituidos por académicos, líderes sociales y autoridades políticas.

Los cursos analizados son MOOC1 "De la desnutrición a la obesidad: un problema de alimentación" ( $1^{\text {a }}$ versión realizada en el año 2017 y $2^{\text {a }}$ versión 2018) y MOOC 2 "Promoción de salud escolar y prevención de la obesidad” de 2018.

\section{Evaluación de los cursos}

La evaluación del aprendizaje consta de una prueba formativa de cuatro preguntas de alternativas, automatizada al final de cada módulo y un trabajo final individual consistente en un breve ensayo. Las pruebas se ponderaron en $40 \%$ y el ensayo en $60 \%$ de la nota final. El ensayo fue de máximo 2.000 caracteres sobre un análisis crítico de una experiencia personal o social relacionada con alguna temática del curso. Este se somete a un proceso de evaluación por pares, con una rúbrica simple de criterios como originalidad de la idea y propósito que desarrolla, formulación de juicios propios, fundamentados con los temas del curso, $\mathrm{o}$ aspectos formales del texto. Cada participante evaluaba dos trabajos asignados al azar y a su vez recibía dos evaluaciones. El curso se aprobó con un puntaje final igual o superior a 50\% del máximo posible, certificado con una Constancia de Honor descargable en PDF. 
El conocimiento de expectativas se realizó mediante una encuesta inicial con las siguientes variables: razones para inscribirse, temores o preocupaciones respecto del curso, horas que espera dedicar, experiencia en cursos MOOC y habilidades digitales.

La evaluación de satisfacción del estudiante se efectuó con una encuesta final que consideraba variables relacionadas con las horas dedicadas semanalmente y dificultades experimentadas; opinión general sobre el curso y materiales, foros, sistema de evaluación, organización del curso y expectativas futuras.

Todas las encuestas han sido validadas o utilizadas previamente en cursos anteriores y consisten en preguntas formuladas con la escala Likert y las siguienes posibilidades: "Muy de Acuerdo", "De Acuerdo", "En Desacuerdo", "Muy en Desacuerdo", "No Sabe/No Responde".

\section{Analisis estadístico}

Se realizó análisis descriptivo cuantitativo de las variables estudiadas, por medio de distribución de frecuencias, promedios y porcentajes, con test de chi-cuadrado para el valor $\mathrm{p}$ de la medición de significancia estadística.

\section{Consideraciones éticas}

El estudio contó con dispensa del documento de Consentimento Informado del Comité Etico Científico del INTA-Universidad de Chile, por razones relativas al carácter de los cursos, beneficios de la evaluación y uso de datos anonimizados de encuestas contestadas en línea en forma voluntaria.

\section{Resultados}

Los contenidos de los cursos se estructuran en 5 módulos, de los cuales 4 son de contenidos y uno de cierre y evaluación, de una semana de duración cada uno (Tabla 1).

\section{Participantes}

El número de participantes efectivos que completaron la evaluación de la primera semana, alcanzó a un total de 17.456 personas: 11.121 en el MOOC1, $1^{\text {a }}$ versión, 2.351 en el MOOC1, 2 ${ }^{\text {a }}$ versión y 3.984 en el MOOC2. Los participantes de los tres cursos tuvieron características similares, con $83,9 \%, 81,5 \%$ y $83,7 \%$ de género femenino, respectivamente. La edad promedio fue $31,8,31,4$ y 31,1 años en cada curso. Se apreciaron diferencias por nivel educacional, con $64,4 \%, 59,3 \%$ y $74,8 \%$ de profesionales o licenciados en los tres cursos. El 64,5\%, 57,6\% y 65,9\% realizaba trabajo remunerado, de los cuales más de un tercio pertenecía al sector salud. La mayoría residía en Chile $(82,9 \%, 79,7 \%$ y $87,2 \%)$ y el resto en otros países de América Latina e incluso hubo algunos casos de Europa y Asia. En todos los casos la prueba de chi cuadrado dio diferencias significativas entre las tres versiones de los cursos, por el gran tamaño de la muestra (Tabla 2).

\section{Encuesta inicial}

\section{Motivaciones y temores para hacer los cursos}

Entre las razones para inscribirse, se observó una tendencia similar en las respuestas de los tres cursos, ubicándose el mayor número de respuestas en las siguientes categorías: a) aprender contenidos

Tabla 1. Contenidos de los Módulos en los Cursos MOOC

\begin{tabular}{|lll|}
\hline Módulos & $\begin{array}{l}\text { De la desnutrición a la obesidad: un problema } \\
\text { de alimentación (MOOC 1, versiones 1 y 2) }\end{array}$ & $\begin{array}{l}\text { Promoción de salud escolar y prevención } \\
\text { de la obesidad (MOOC 2) }\end{array}$ \\
\hline Módulo 1 & Alimentación, salud y calidad de vida & Salud y calidad de vida \\
\hline Módulo 2 & Alimentación en los primeros años de vida & $\begin{array}{l}\text { Políticas relacionadas con la promoción de salud } \\
\text { escolar }\end{array}$ \\
\hline Módulo 3 & Alimentación y desarrollo escolar & Alimentación saludable y actividad física \\
\hline Módulo 4 & Recomendaciones para una alimentación saludable & $\begin{array}{l}\text { Prácticas y desafíos de la comunidad educativa en la } \\
\text { prevención de la obesidad y promoción de la salud }\end{array}$ \\
\hline
\end{tabular}


Tabla 2. Perfil de los participantes en los Cursos MOOC

\begin{tabular}{|c|c|c|c|c|}
\hline \multirow[t]{2}{*}{ Total } & \multirow{2}{*}{$\begin{array}{c}\text { De la Desnutrición a } \\
\text { la Obesidad } \\
\text { (MOOC 1, 1a versión) } \\
\text { n (\%) } \\
11.121(100)\end{array}$} & \multirow{2}{*}{$\begin{array}{c}\text { De la Desnutrición a } \\
\text { la Obesidad } \\
\text { (MOOC 1, 2 } 2^{\text {a versión) }} \\
\text { n (\%) } \\
2.351(100)\end{array}$} & \multicolumn{2}{|c|}{$\begin{array}{l}\text { Promoción Salud } \\
\text { Escolar y Prevención } \\
\text { Obesidad (MOOC 2) }\end{array}$} \\
\hline & & & $\begin{array}{c}n(\%) \\
3.984(100)\end{array}$ & Valor $\mathbf{p}$ \\
\hline $\begin{array}{l}\text { Sexo } \\
\text { Mujeres } \\
\text { Hombres }\end{array}$ & $\begin{array}{l}9.336(83,95) \\
1.785(16,05)\end{array}$ & $\begin{array}{r}1.916(81,5) \\
435(18,5)\end{array}$ & $\begin{array}{c}3.335(83,7) \\
649(16,2)\end{array}$ & 0,014 \\
\hline $\begin{array}{l}\text { Edad años promedio } \\
\text { DS (Rango) }\end{array}$ & $\begin{array}{c}31,8 \\
9,57(13-84)\end{array}$ & $\begin{array}{c}31,4 \\
8,95(14-78)\end{array}$ & $\begin{array}{c}31,1 \\
8,64(10-90)\end{array}$ & $<0,0001$ \\
\hline \multicolumn{5}{|l|}{ Nivel educacional } \\
\hline $\begin{array}{l}\text { Doctor PHD/Magister } \\
\text { Licenciado/profesional universitario } \\
\text { Técnico-profesional } \\
\text { Educ. media/secundaria completa } \\
\text { Educación básica/primaria } \\
\text { completa o incompleta }\end{array}$ & $\begin{array}{c}867(7,8) \\
7.162(64,4) \\
1.079(9,7) \\
1.702(15,3) \\
311(2,8)\end{array}$ & $\begin{array}{c}181(7,7) \\
1394(59,3) \\
310(13,2) \\
447(19) \\
19(0,8)\end{array}$ & $\begin{array}{c}315(7,9) \\
2980(74,8) \\
299(7,5) \\
383(9,6) \\
8(0,2)\end{array}$ & \\
\hline \multicolumn{5}{|l|}{ Actividad } \\
\hline Trabaja & $\begin{array}{c}7.173(64,5) \\
\text { (38 salud, } 22 \text { educación, } \\
40 \text { otros sectores) }\end{array}$ & $\begin{array}{c}1.354(57,6) \\
(32,1 \text { salud, } 16,5 \\
\text { educación, } 51,4 \text { otros } \\
\text { sectores })\end{array}$ & \multicolumn{2}{|c|}{$\begin{array}{c}\text { (39,7 salud, } 33,7 \text { educación } \\
26,6 \text { otros sectores) }\end{array}$} \\
\hline Estudia & $\begin{array}{c}2.102(18,9) \\
\text { (65 área salud, } 14 \\
\text { educación y cs. sociales, } \\
21 \text { otras áreas) }\end{array}$ & $\begin{array}{c}459(19,5) \\
\text { (no se incluyó la } \\
\text { pregunta área de } \\
\text { estudio) }\end{array}$ & \multicolumn{2}{|c|}{$\begin{array}{c}940(23,6) \\
\text { (67 área salud, } 21,8 \\
\text { educación y cs. sociales, } \\
11,2 \text { otras áreas) }\end{array}$} \\
\hline Desempleado & $1.779(16)$ & $520(22,1)$ & \multicolumn{2}{|c|}{$418(10,5)$} \\
\hline Jubilado, otros & $67(0,6)$ & $19(0,8)$ & \multicolumn{2}{|c|}{$8(0,2)$} \\
\hline \multicolumn{5}{|l|}{ País de residencia } \\
\hline Chile & $9.217(82,8)$ & $1.857(79,7)$ & $3.474(87,2)$ & $<0,0001$ \\
\hline Otros países de residencia & $\begin{array}{c}1.904(17,2) \\
\text { (6,1 Ecuador, 2,3 México, } \\
\text { 2,3 Perú, 1,3 Colombia, } \\
\text { 1,3 Argentina, 3,9 otros } \\
\text { países de Europa y Asia) }\end{array}$ & $\begin{array}{c}494(20,3) \\
\text { (6,7 Ecuador, 3,2 México, } \\
\text { 2,8 Perú, 2,5 Colombia, } \\
\text { 1,2 Argentina, 3,9 otros } \\
\text { países) }\end{array}$ & \multicolumn{2}{|c|}{$\begin{array}{l}510(12,8) \\
\text { (4,1 Ecuador, 1,9 Perú, 1,2 } \\
\text { México, 1,1 Argentina, 1,0 } \\
\text { Colombia, 3,5 otros países) }\end{array}$} \\
\hline Regiones de pertenencia en Chile & $3.754(40,7)$ & $589(31,7)$ & $1.213(34,9)$ & $<0,0001$ \\
\hline $\begin{array}{l}\text { Región Metropolitana } \\
14 \text { regiones }\end{array}$ & $5.463(59,3)$ & $1.268(68,3)$ & \multicolumn{2}{|c|}{$2.262(65,1)$} \\
\hline
\end{tabular}

o herramientas que pueda aplicar en mi trabajo o estudios $(61,9 \%)$; b) gusto o interés personal de aprender nuevos conocimientos (53,1\%), y c) aprender contenidos o herramientas que pueda aplicar en mi vida cotidiana (39,3\%).

Respecto a los principales temores, existió coincidencia al señalar que la principal preocupación era no disponer de tiempo (42\%). El 84\% manifestó estar decidido a terminar el curso y $27 \%$ de dedicar al estudio 1 a 2 h y $30 \%$ de 3 a 4 h a la semana.

\section{Habilidades digitales}

La tendencia fue similar en los tres cursos: $50 \%$ señaló que podía usar eficazmente algunas aplicaciones o programas de uso común (procesa- 
dor de textos, planillas electrónicas, exploradores de internet o redes sociales) y $32 \%$ usaba varias aplicaciones o programas diferentes, característica consistente con $53 \%$ que refirió tener experiencia en cursos MOOC.

\section{Encuesta final}

\section{Evaluación}

La evaluación final fue altamente satisfactoria en los tres cursos. Ante la pregunta ¿estoy satisfecho con el curso? las respuestas fueron $85 \%, 92 \%$ y 99\% "Muy de Acuerdo" y "De Acuerdo", lo que también dio muy significativo por el tamaño de la muestra (Tabla 3).

Los indicadores de evaluación fueron muy positivos en relación a la entrega de herramientas conceptuales y metodológicas, la calidad de videoclases y entrevistas, lecturas y materiales complementarios, con cifras entre $85 \%$ y $98 \%$. La valoración del aporte de los foros fue menor, fluctuando entre $62 \%$ y $75 \%$ : entre $30 \%$ y $52 \%$ de los estudiantes señalaron haber participado activamente en ellos.

La evaluación semanal realizada en base a pruebas de conocimientos fue considerada adecuada y relevante ( $81 \%$ al $97 \%$ ). Entre $73 \%$ y $85 \%$ opinaron que la evaluación final de pares funcionó correctamente y entre $80 \%$ y $89 \%$ consideró que los criterios para evaluar a sus compañeros fueron claros y pertinentes. Finalmente, entre 64\% y $74 \%$ de los estudiantes consideraron que la evaluación recibida fue justa y fundamentada.

Entre $86 \%$ y $99 \%$ opinó que la organización y programa del curso fue clara y oportuna y una cifra menor (49\% y 58\%) señaló que pudo resolver sus dudas. Las expectativas de continuar vinculados con sus compañeros y docentes fluctuó entre $84 \%$ y $97 \%$. Se observó un alto interés para participar en otros cursos MOOC, el que varió de $87 \%$ a $98 \%$.

$\mathrm{Al}$ consultar por los problemas que debió enfrentar durante el desarrollo de los cursos, 50\% señaló no haber tenido ninguna dificultad; 35\% tuvo falta de tiempo, $11 \%$ problemas de conexión a internet o computador y $3,9 \%$ señaló problemas personales o laborales.

La evaluación final fue aprobada por 8.878 participantes, quienes recibieron una "Constancia de Honor". La tasa de finalización exitosa se calculó en base a dos criterios del universo grupal: uno considerando el número de participantes inscritos y el otro en relación al número de participantes efectivos, alcanzando $26,84 \%$ de los inscritos y $50,85 \%$ de los que efectivamente hicieron el curso (Tabla 4).

\section{Discusión}

Estos cursos MOOC pueden llegar a miles de personas sin importar el lugar del mundo en el cual se encuentran ${ }^{19}$. Esta masividad es más importante si consideramos que se desarrollaron antes de la pandemia COVID-19, en la cual se instalaron cuarentenas prologadas que obligaron al sistema educativo y productivo a cambiar sus métodos de educación y trabajo por metodologías a distancia, las que en el futuro van a ser modalidades preferentes en lo educativo. Las instituciones de educación superior han debido innovar sus programas docentes, junto con acelerar el proceso de transformación digital de la educación regular presencial ${ }^{20,21}$. Esto ha sido particulamente critico en el ámbito médico y de la salud, que ha debido instalar una educación de emergencia online, para capacitar a quienes trabajan en el manejo de la pandemia COVID-1922,23. Los cursos MOOC pueden llegar a audiencias mucho mayores, muy diversas y no alcanzables físicamente con los métodos presenciales, lo cual va a contribuir a la democratización del conocimiento. Esto quedó demostrado al tener participantes de todas las regiones del país y de otros países, incluidos de Europa y Asia.

Hubo una alta tasa de finalización exitosa, en cifras promedios $50,85 \%$ de los que efectivamente terminaron y aprobaron en los 3 cursos, cifras superiores a lo observado en otras experiencias internacionales y nacionales. Por ejemplo, Ale$\operatorname{man}^{24}$ realiza un análisis comparativo de 12 cursos MOOC de distintas áreas académicas de universidades latinoamericanas pioneras en educación a través de la plataforma Coursera, señalando que solo un curso tuvo eficiencia terminal de $22,35 \%$, lo que califica como atípico, ya que los otros cursos tuvieron valores cercanos a $6,5 \%$ de finalización, que es el promedio registrado en la literatura. En cursos con matrícula menor se han reportado tasas de finalización de alrededor de $30 \%$, como señala Benet ${ }^{17}$ en un MOOC de educación y psicología, en el cual de 330 inscritos finalizaron $110(33 \%)$. 


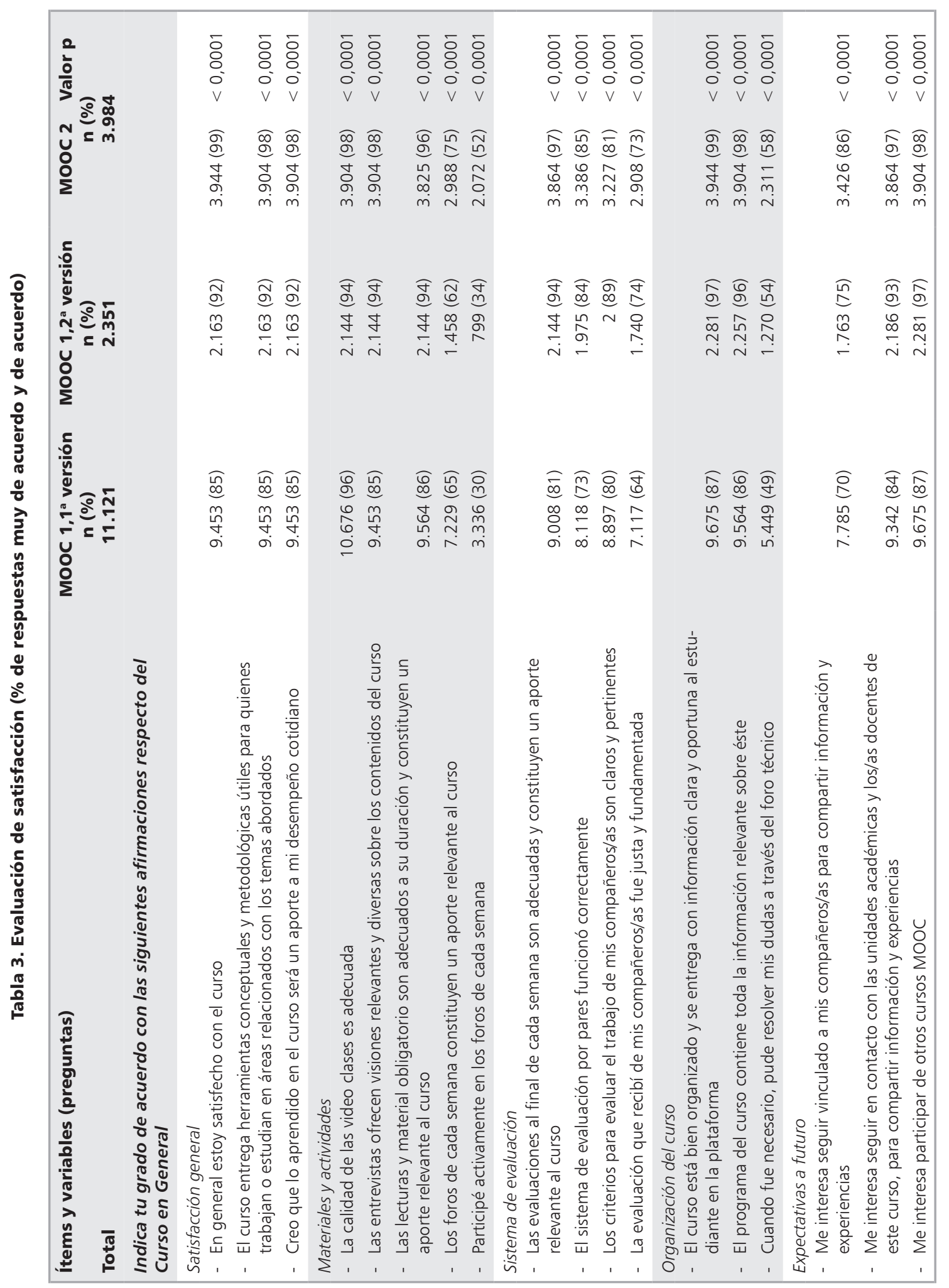


Tabla 4. Tasa de finalización en relación al número (n) de participantes inscritos y efectivos de los cursos MOOC

\begin{tabular}{|lccccc|}
\hline Nombre del curso (Versión) & $\begin{array}{c}\mathbf{n} \text { de } \\
\text { participantes } \\
\text { inscritos }\end{array}$ & $\begin{array}{c}\mathbf{n} \text { de } \\
\text { participantes } \\
\text { efectivos } \\
\text { (evaluación } \\
\mathbf{1}^{\mathbf{a}} \text { semana) }\end{array}$ & $\begin{array}{c}\mathbf{n} \text { de } \\
\text { constancias } \\
\text { de honor } \\
\text { (evaluación } \\
\text { final) }\end{array}$ & $\begin{array}{c}\text { Finalización } \\
\text { exitosa } \\
\text { \% }\end{array}$ & $\begin{array}{c}\text { Aprobación } \\
\text { participantes } \\
\text { inscritos }\end{array}$ \\
$\begin{array}{l}\text { participantes } \\
\text { efectivos }\end{array}$ \\
$\begin{array}{l}\text { De la desnutrición a la obesidad: } \\
\text { un problema de alimentación } \\
\text { (MOOC 1, 1a versión) }\end{array}$ & 16.268 & 11.121 & 4.472 & 27,5 & 40,2 \\
$\begin{array}{l}\text { De la desnutrición a la obesidad: } \\
\text { un problema de alimentación } \\
\text { (MOOC 1, 2a versión) }\end{array}$ & 6.769 & 2.351 & 2.008 & 29,66 & 85,4 \\
\hline $\begin{array}{l}\text { Promoción de la Salud Escolary Pre- } \\
\text { vención de la Obesidad (MOOC 2) }\end{array}$ & 10.045 & 3.984 & 2.398 & 23,9 & 60,2 \\
\hline \begin{tabular}{l} 
Total \\
\hline
\end{tabular} & 33.082 & 17.456 & 8.878 & 26,84 & 50,85 \\
\hline
\end{tabular}

En la evaluación de ocho cursos U Abierta de la Universidad de Chile el 2016 se obtuvo una tasa de finalización exitosa de 27,4\%, considerando como universo las personas que realizaron al menos una actividad, criterio menos exigentes al empleado en este estudio. En cuanto a la satisfacción, fue superior a otros estudios internacionale ${ }^{24}$ y similar a otros cursos U Abierta ${ }^{18}$.

La evaluación de estos cursos MOOC es reciente, ya que solo desde el 2015 aparecieron las primeras publicaciones ${ }^{25,26}$. A nivel nacional se encontró solo una publicación en cursos de ingeniería, educación y administración ${ }^{27}$ y en el ámbito internacional la mayoría está comunicada en libros de actas de congresos ${ }^{28}$. Muchos autores coinciden en que la evaluación es uno de los aspectos más deficitarios de estos cursos masivos, entendida como un paso más del proceso educativo. Existe controversia sobre mecanismos alternativos de evaluación de pares y test o pruebas. En este caso se utilizaron ambas estrategias, obteniéndose un buen resultado y satisfacción de los participantes.

Estos cursos MOOC son la primera experiencia desarrollada en el ámbito de la salud y nutrición en Chile y America Latina. De acuerdo a los resultados, destacamos su importancia para educar en forma masiva y flexible, sin requisito de ingreso ni costo para los participantes. Al mismo tiempo, permiten a las universidades generar espacios de aprendizaje con las comunidades, que contribuyan al desarrollo de estilos de vida y entornos mas saludables para prevenir la obesidad y las enfermedades crónicas no transmisibles.

Como fortaleza destacamos la posibilidad de evaluar cursos masivos a distancia con la información directa que aporta la plataforma, sin necesidad de estudios específicos que requieran gasto y complejidades extras, lo cual puede servir de modelo para evaluar futuros cursos. Como debilidad, este estudio no contó con metodologías cualitativas que permitiesen conocer en profundidad las necesidades de formación, la calidad de las metodologías empleadas, los aprendizajes obtenidos y otros aspectos educativos necesarios para formular futuros programas, lo cual queda pendiente para otros estudios.

\section{Conclusiones}

Esta modalidad de cursos MOOC abiertos, masivos, en línea y gratuitos es la primera experiencia desarrollada en salud y nutrición para prevenir la obesidad en Chile. De acuerdo a los resultados, destacamos su importancia para llegar a todo el país, América Latina y países de otras regiones para educar en forma masiva y flexible para contribuir al cambio de conducta que permita prevenir la obesidad y las enfermedades crónicas no transmisibles. 
Agradecimientos: Este trabajo fue apoyado por los Programas EduAbierta y UAbierta de la Universidad de Chile.

\section{Referencias}

1. Vio F, Kain J. Descripción de la progresión de la obesidad y enfermedades relacionadas en Chile. Rev Med Chile 2019; 147: 1159-65.

2. NCD Risk Factor Collaboration (NCD-RisC). Worldwide trends in body-mass index, underweight, overweight, and obesity from 1975 to 2016: a pooled analysis of 2416 population-based measurement studies in 128.9 million children, adolescents, and adults. Lancet 2017; 390: 2627-54.

3. Agencia de Calidad de la Educación. Estudio Nacional Educación Física 2015 Disponible en: http://archivos. agenciaeducacion.cl/Informe_Nacional_EducacionFisica2015.pdf [Consultado el 17 de junio de 2020].

4. Junta Nacional de Auxilio Escolar y Becas (JUNAEB). Mapa Nutricional 2019. Disponible en: https://www. junaeb.cl/wp-content/uploads/2013/03/Mapa-Nutricional-2019-1.pdf [Consultado el 17 de junio de 2020].

5. Programa de las Naciones Unidas para el Desarrollo (PNUD). Desigualdades Orígenes, cambios y desafíos de la brecha social en Chile 2017. Disponible en: https://www.cl.undp.org/content/chile/es/home/library/ poverty/desiguales--origenes--cambios-y-desafios-dela-brecha-social-en-.html [Consultado el 17 de junio de 2020].

6. Sánchez H, Albala C. Desigualdades en salud: mortalidad del adulto en comunas del Gran Santiago. Rev Méd Chile 2004; 132: 453-60.

7. Organización Mundial de la Salud. Facts Sheets. Obesity and overweight 2017. Disponible en: https://www.who. int/news-room/fact-sheets/detail/obesity-and-overweight [Consultado el 17 de junio de 2020]

8. Vio F. De la Obesidad al Coronavirus: una visión desde la salud pública. Editorial Universidad de Talca, Primera Edición, Talca - Chile, julio de 2020. Salesianos Impresores S.A.

9. Swinburn BA, Kraak VI, Allender S, Atkins VJ, Baker PI, et al. The Global Syndemic of Obesity, Undernutrition, and Climate Change: The Lancet Commission report. www.thelancet.com Published online January 27, 2019.

10. Salinas J, Muñoz C, Albagli A, Araya G, Vio F. The contribution of distance education to health promotion in Chile. Health Promot Int. 2017; 32: 913-21.

11. Salinas J, Muñoz C, Albagli A, Vio F. Evaluación de un programa de educación a distancia en Promoción de Salud para la Atención Primaria en Chile. Rev Med Chile 2014; 142: 184-92.

12. Salinas J, Vio F. Educación a distancia en promoción en salud: Una experiencia innovadora. Rev Med Maule 2011: 28:10-6.

13. Mercado del Collado R. Cursos masivos abiertos en línea: oportunidad o amenaza. Universidades UDUAL 2016; 70: 53-68. Disponible en: https://www.redalyc.org/ pdf/373/37348529005.pdf [Consultado el 17 de junio de 2020].

14. Vinader R, Abuín N. Nuevos modelos educativos: los MOOCs como paradigma de la formación online. Historia y Comunicación Social 2013; 18: 801-14.

15. Torres D, GaGo D. Los MOOCS y su papel en la creación de comunidades de aprendizaje y participación. RIED 2014; 17: 13-34.

16. Cabero J. Visiones educativas sobre los MOOC. RIED 2015; 18: 39-60.

17. Benet A, Sanahuja A, García I, Nieto R. Nuevos horizontes formativos: una experiencia del MOOC como recurso en la formación continua. Apert. (Guadalaj. Jal.) 2018; 10: 88-103. Disponible en: http:// www.scielo.org.mx/scielo.php?script=sci_arttext\&pi$\mathrm{d}=$ S1665-61802018000100088\&lng=es\&nrm=iso [Consultado el 17 de junio de 2020].

18. Cano ME, Fernández M, Crescenzi L. Cursos en Línea Masivos y Abiertos: 20 expertos delinean el estado de la cuestión. RELATEC 2015; 14: 2537.

19. Callejo J, Agudo Y. MOOC: valoración de un futuro RIED 2018; 21: 219-41.

20. Ruiz C. El MOOC: ¿un modelo alternativo para la educación universitaria? Apert. (Guadalaj.Jal.) 2015; 7: 86-100. Disponible en: http://www.scielo.org.mx/scielo.php?script $=$ sci_arttext \&pi$\mathrm{d}=\mathrm{S} 1665-61802015000200086 \& \operatorname{lng}=\mathrm{es} \& \mathrm{nrm}=\mathrm{iso}$ [Consultado el 17 de junio de 2020].

21. Pardo H, Cobo C. Expandir la universidad más allá de la enseñanza remota de emergencia. Ideas hacia un modelo híbrido post-pandemia. 2020. Outliers School. Barcelona. Disponible en: http://outliersschool.net/project/ universidadpostpandemia/ [Consultado el 17 de junio de 2020].

22. Challenges to medical education at a time of physical distancing. The Lancet Online June 11, 2020. Disponible en: https://doi.org/10.1016/ S0140-6736(20)31368-4 [Consultado el 12 de junio de 2020].

23. Crawford J, Butler-Henderson K, Rudolph J, Malkawi B, Glowatz M, Burton R, et al. COVID-19: 20 countries' higher education intra-period digital pedagogy responses. Journal of Applied Learning \& Teaching 2020; 3: 1-20. Disponible en: https://journals.sfu.ca/jalt/index. 
php/jalt/article/view/191/163 [Consultado el 17 de junio de 2020].

24. Alemán L, Sancho T, Gómez G. Análisis de un curso en línea masivo y abierto (MOOC) con una eficiencia terminal atípica. Revista Internacional De Tecnología, Ciencia y Sociedad 2016; 5 (1): 91-101.Disponible en: https://doi.org/10.37467/gka-revtechno.v5.460 [Consultado el 17 de junio de 2020].

25. Sánchez M, González V, Prendes M. Los MOOC y la evaluación del alumnado: revisión sistemática (20122016) @tic.revista d'innovació educativa Universidad de Valencia 2017; 18: 65-73.
26. González A, Carabantes D. MOOC: medición de satisfacción, fidelización, éxito y certificación de la educación digital. RIED 2017; 20: 105-23. DOI: http://dx.doi. org/10.5944/ried.20.1.16820

27. Maldonado J, Pérez M, Kizilcec R, Morales N, Muñoz J. Mining theory-bases patterns from Big data: Identifying self-regulated learning strategies in MOOC. Computers in Human Behavior 2018; 80: 179-96.

28. Prieto M, Pech S, Zapata A. Eds. Tecnología y Aprendizaje. Avances en el Mundo Académico Hispano. Editorial CIATA.org 2017, ISBN: 978-84-697-2772-0 Ciudad Real, España. 